\title{
LA CONSTRUCTION DU DISCOURS ANTI-DJIHADISTE SUR INTERNET : ENJEUX, THÉMATIQUES, PROCÉDÉS
}

\section{INTRODUCTION : OBJECTIF ET CHAMP D'ÉTUDE}

Le djihad, tel que conceptualisé et présenté par les islamistes radicaux, est une guerre qui a pour but d'anéantir les « infidèles », ceux qui ne croient pas en Allah, Dieu de l'islam. N'ayant pas de respect pour la vie humaine, les djihadistes imposent la terreur et l'assassinat de civils par des attentats-suicide. D'un côté, ils obtiennent ainsi, de façon délibérée, le choc psychologique et l'angoisse qu'ils espèrent provoquer, mais d'un autre, leurs actes suscitent au sein des populations visées une réaction de résistance, naturelle pour qui se sent constamment menacé. Cette résistance s'exprime, entre autres, dans le discours anti-djihadiste présent sur Internet par différents phénomènes communicationnels à travers lesquels les citoyens ordinaires « combattent » les islamistes en les ridiculisant en vue de les disqualifier.

L'objectif de cette contribution est triple. D'une part, il s'agit de tenter de montrer par quels mécanismes concrets se construit ce discours. À travers quels procédés et selon quels principes ? Quelle approche privilégier comme cadre pour son analyse ? D'autre part, il est intéressant de savoir quelles sont ses thématiques et sa finalité. Quel imaginaire socio-discursif se profile-t-il derrière ce discours ? Y est-il uniquement question de discréditer l'ennemi islamiste pour se défendre d'une réalité angoissante ? Ou y entrevoit-on l'éthos discursif de qui le construit, une image que le locuteur tient absolument à donner de lui-même dans ces messages ? Enfin - pour rejoindre les préoccupations de ce numéro de la revue - il sera question de la place que des termes familiers, populaires et argotiques occupent dans ce discours et de la fonction qu'ils y remplissent.

\section{CADRE THÉORIQUE}

Le discours anti-djihadiste des internautes est une réponse aux attaques terroristes. Son apparition dans le cyberespace témoigne de l'existence d'un conflit entre deux univers de valeurs différents, deux visions du monde paraissant inconciliables. Ce conflit, une fois transposé par les internautes au sein du web 2.0, a commencé à se dérouler devant un vaste public et à se définir, en corollaire, par sa double adresse : il vise simultanément sa cible, i.e. les djihadistes, et un tiers plus ou moins repérable, le public devant lequel la cible doit être discréditée. Ce discours est ainsi une sorte de guerre où le locuteur (collectif) essaie de s'attirer des alliés par une argumentation visant à faire partager

ages@wp.pl 
sa vision du monde, tout en mettant en cause celle de l'ennemi. Notre étude s'inscrit donc naturellement dans le cadre théorique de l'analyse argumentative du discours, telle qu'envisagée par Ruth Amossy (2006).

Il reste à se demander quelles stratégies adoptent les internautes pour atteindre leur but, en reprenant les mots d'Amossy (2011 : 19), pour « concrétiser [leur] projet de persuasion » et « infléchir des façons de voir et d'interpréter le réel » et si ces stratégies sont à la hauteur de leurs enjeux. Nous nous efforcerons de montrer qu'ils déploient, en vue de faire partager leur rejet de la vision du monde de l'ennemi, une argumentation visant à faire entrer le public en connivence avec eux. Connivence qu'ils créent, comme on le verra, par recours au phénomène de l'humour verbo-iconique.

\section{CORPUS SOUMIS À L'ANALYSE}

Le corpus soumis à notre analyse, qui ne se veut ni systémique ni exhaustive, se compose d'échantillons du discours anti-djihadiste tels que les mèmes numériques, caricatures et dessins français, belges et polonais glanés çà et là sur la toile, qui sont des exemples de phénomènes viraux humoristiques. Il s'agit de ce que R. Jakobson (1971) appelait les syncretic messages ${ }^{1}$ (messages syncrétiques), basés sur une combinaison des systèmes sémiotiques différents. Rapidement relayés sur Internet, ils acquièrent une grande popularité en peu de temps, ce en quoi ils semblent être un excellent outil de lutte discursive contre le djihad.

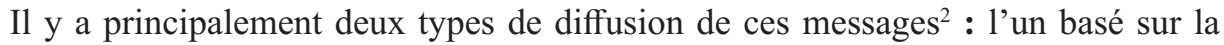
réplication, l'autre sur la variation. La première forme relaie, sans le modifier, le matériel viral - dans notre cas ce sont les dessins et les caricatures; la seconde intègre des différences dans le matériel, et ce sont les mèmes numériques, qui se composent le plus souvent d'un élément stable, une photo, et d'un élément variable, un court texte qui canalise le sens du message iconique. Comme l'explique Maude Bonenfant (2014) :

Le concept de mème est [...] défini comme un élément de code culturel qui est reconnaissable et reproductible. Il provient du meme de Richard Dawkins (1976), concept créé par la contraction des mots gène et mimésis (imitation). Dawkins établit un parallèle entre le biologique (nature) et l'information (culture) afin de comprendre comment une information peut circuler et muter comme un gène. Il pose ainsi une équivalence structurelle entre le code génétique et le code culturel : tout comme les gènes se répliquent et mutent, il y aurait dissémination des informations culturelles par imitation et transformation.

Dans ce passage éclairant il n'est pas question du caractère ludico-humoristique des mèmes, mais celui-ci nous semble en être un critère définitoire. L'humour dont sont imprégnés les mèmes et les dessins analysés est le premier mot-clé de l'analyse

1 « The study of communication must distinguish between homogeneous messages which use a single semiotic system and syncretic messages based on a combination or merger of different sign patterns » (Jakobson 1971 : 705).

2 Voir M. Bonenfant (2014). 
effectuée ci-après. Dans le cas qui nous occupe, ce phénomène rend possible l'argumentation contre la cible du discours, sans même mentionner la possibilité qu'il offre à l'énonciateur de préserver son équilibre émotionnel dans une situation difficile comme celle des attentats.

\title{
4. HUMOUR ET CONNIVENCE
}

Selon Patrick Charaudeau (2006: 22), la nature de l'acte de discours humoristique est déterminée par une relation triadique caractérisant la situation d'énonciation :

\begin{abstract}
L'acte humoristique comme acte d'énonciation met en scène trois protagonistes : le locuteur, le destinataire et la cible. Le locuteur est celui qui, à l'intérieur d'une certaine situation de communication, produit l'acte humoristique [...]. Le problème qui se pose à lui est celui de sa légitimité, de ce qui l'autorise à produire dans cette situation un acte humoristique. Car ne produit pas un acte humoristique qui veut, sans tenir compte de la nature de son interlocuteur, de la relation qui s'est instaurée entre eux, des circonstances dans lesquelles il est produit. Selon les cas, un acte humoristique peut blesser l'autre ou le rendre complice. Le locuteur doit donc avoir vis-à-vis de son interlocuteur une position qui à la fois légitime son énonciation humoristique et justifie, voire explique, le jeu langagier auquel il se livre à propos de tel thème, en visant telle cible.
\end{abstract}

Quelle est la position du locuteur envers son interlocuteur dans le cas du discours anti-djihadiste sur Internet? Légitime-t-elle l'énonciation humoristique ? Comme nous allons le voir, une grande partie des messages analysés bousculent les frontières du tolérable, ils sont caustiques, virulents, vulgaires. En imprégnant son message d'un type d'humour qui peut être difficile à accepter de par son caractère transgressif, le locuteur risque facilement, dit Charaudeau (op. cit., p. 25), de « voir se retourner la mise en cause du monde contre sa propre personne et de faire l'objet d'une sanction ». Et pourtant, son objectif est de faire de son interlocuteur un complice, de produire sur l'auditoire un effet de connivence qui vise son adhésion à la disqualification de la cible de son discours.

Différents paramètres légitiment, croyons-nous, sa manière de dire. Nous n'en citerons que deux. Tout d'abord, le contexte situationnel : les messages en question sont émis en réaction aux attentats, qui ont un impact terrifiant sur les gens. L'humour, même le plus agressif, semble être bien peu de chose en comparaison de la perte de la vie humaine, comme l'illustre de manière bien ironique ce dessin (1) de Delize.

Ensuite - et c'est là le facteur primordial - même si du fait de la spécificité du canal de communication le locuteur ne connaît pas son auditoire et ne peut que se l'imaginer, il adresse ses messages à un destinataire qui, par définition et à coup sûr, fait partie des « siens » lesquels, indépendamment du type d'humour, blessant ou non, vont l'accepter et adhérer à la critique qu'il véhicule. Qu'il s'agisse certainement des « siens », en témoigne la double structure des messages que nous avons soumis à l'analyse. Ils se divisent en deux groupes : d'un côté, nous avons ceux qui se focalisent sur la cible du 


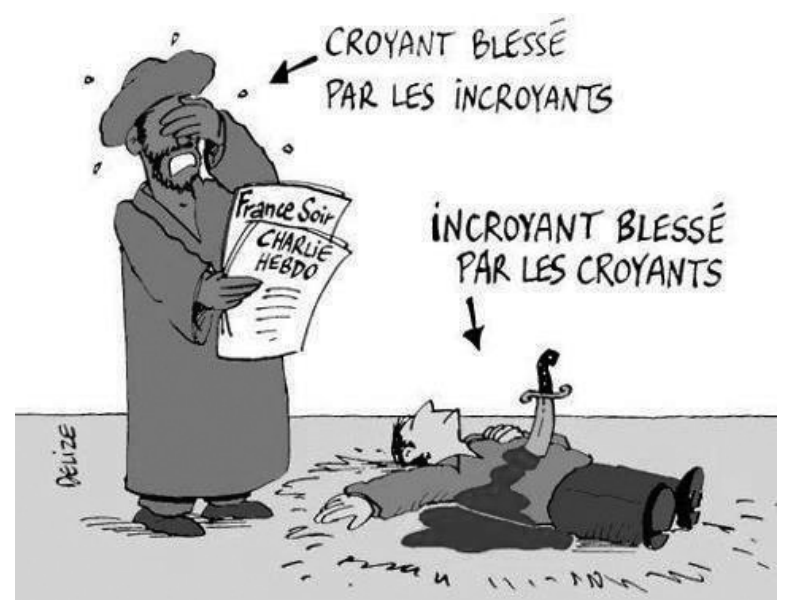

Image 1. Auteur : Dominique Lizambard (Delize)

Source : https://www.bbc.com/news/in-pictures-30712925

discours et de l'autre, les messages autocentrés, polarisés sur le locuteur même qui lutte contre l'ennemi en construisant son propre éthos discursif pour souligner que lui et - soulignons-le bien - sa communauté, se trouvent aux antipodes de l'ennemi dont la vision du monde est ainsi mise en cause. Cette construction bipartite du discours le situe dans le cadre de la relation dyadique entre le Soi et l'Autre.

\section{5. « MÊMÉITÉ » ET ALTÉRITÉ}

Les internautes caricaturent l'identité des djihadistes pour protéger la leur, mus par le désir de renforcer le sentiment communautaire soudant leur groupe et les situant ensemble « de l'autre côté du mur ». Dans les messages du premier type, concentrés sur la cible du discours, on observe une forte mise en question du djihad, mais le locuteur s'en prend aussi à l'islam en général. Est ainsi moquée " la guerre sainte » des islamistes, mais aussi leur religion et ses symboles, tout comme les stéréotypes que le locuteur s'en fait. Celui-ci émet également des messages où il ne fait référence indirecte à l'Autre qu'en se présentant implicitement comme diamétralement opposé à lui. Tout cela montre que le discours anti-djihadiste se construit autour des principes de " mêméité » et d'altérité, ce dont rend compte Charaudeau (2009) en ces mots :

Ce n'est qu'en percevant l'autre comme différent que peut naître la conscience identitaire. La perception de la différence de l'autre constitue d'abord la preuve de sa propre identité [...]. [C]ette différence représente une menace pour le sujet. [...]. C'est pourquoi la perception de la différence s'accompagne généralement d'un jugement négatif. Il y va de la survie du sujet. [...] Lorsque ce jugement se durcit et se généralise, il devient ce que l'on appelle traditionnellement 
un stéréotype, un cliché, un préjugé. Ne méprisons donc pas les stéréotypes. Ils sont une nécessité. Ils constituent d'abord une protection, une arme de défense contre la menace que représente l'autre dans sa différence [...]. Ces jugements négatifs ont une conséquence fâcheuse : en jugeant l'autre négativement on protège son identité, on caricature celle de l'autre, et du même coup la sienne, et l'on se persuade que l'on a raison contre l'autre.

Comme toujours, la peur suscitée par la différence entre le Soi et l'Autre engendre des malentendus dus à la méconnaissance de la culture (et de la religion) de l'Autre, d'où l'apparition de clichés et stéréotypes simplistes par définition. Nous analyserons en premier lieu les messages qui s'en nourrissent, pour passer ensuite à ceux polarisés sur le Soi.

\section{ANALYSE DES MESSAGES FOCALISÉS SUR L'AUTRE}

L'analyse des thématiques prises pour cible dans le discours anti-djihadiste révèle que les messages du premier type font incontestablement l'amalgame entre terroriste et musulman, en ceci qu'ils s'attaquent non seulement aux actions des islamistes radicaux, mais aussi aux symboles stéréotypés de l'islam. Un linguiste ou un analyste du discours n'a pourtant pas à juger du bien-fondé d'un tel mélange, mais doit analyser plutôt les causes possibles d'une telle attitude et les thématiques attaquées.

Les traits de caractère et les défauts attribués aux islamistes (en particulier l'ignorance, le manque d'intérêt pour la culture, la peur de réfléchir, l'aveuglement idéologique, l'hypocrisie) constituent logiquement la cible du discours anti-djihadiste. Mais du fait des questions d'identité, de " mêméité » et d'altérité, ce sont aussi les éléments que l'Occident a stéréotypés et érigés en symboles islamiques (la burqa, la barbe, la pratique du suicide par explosion pour tuer les infidèles et être récompensé par 72 vierges au paradis, la formule Allahu akbar !).

\section{1. La burqa}

Il est intéressant de constater que la burqa (ou le voile que l'on désigne abusivement par ce nom ${ }^{3}$ ) est, curieusement, le symbole islamique que les internautes attaquent le plus souvent. Ils tournent en dérision cet emblème en relayant sur la toile les dessins et les mèmes comparant les musulmanes qui s'en recouvrent à différents objets, p. ex. aux parasols, comme dans le mème polonais suivant ${ }^{4}$ :

3 Les messages prenant pour cible cet élément illustrent très bien le processus de création de stéréotypes. On commence à l'aide de quelques bribes d'informations, ensuite on simplifie et on généralise outre-mesure et finalement on forge un stéréotype très souvent erroné. Le fait de désigner tout vêtement de femmes musulmanes par le mot burqa, en soit-il une ou non, en est un bon exemple.

4 « J'ai passé une demi-heure avec trois musulmanes en parlant avec elles de leur culture, de leur religion et de leurs mœurs, et à la fin le barman m'a appris que c'étaient des parasols ». 


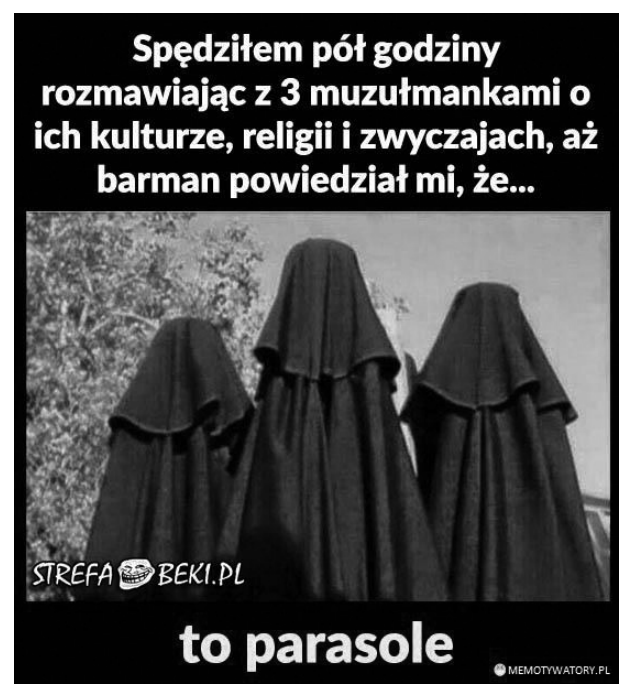

Image 2. Auteur inconnu

Source : https://memotywatory.pl/529658,kiedy-impreza-jest-zbyt-gruba

ou à un sac à déchets, comme dans $(3)^{5}$ :

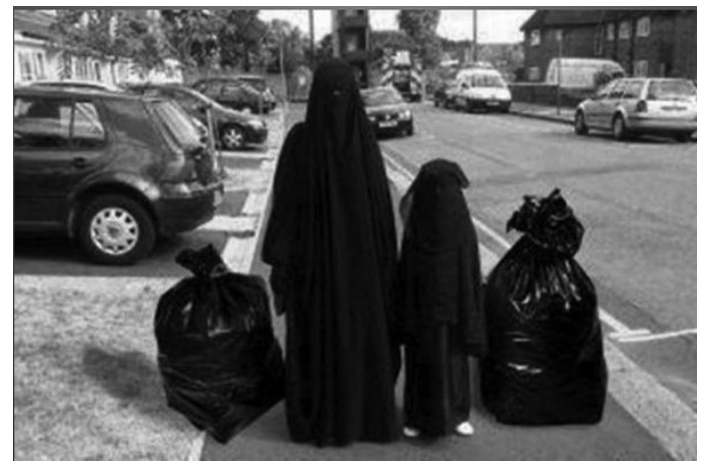

Image 3. Auteur inconnu

Source : http://chantouvivelavie.centerblog.net/8702-Humour-burqa

Ces trois messages réduisent les musulmanes à des objets, procédé que l'on pourrait appeler " chosification » de l'être humain. Le même procédé est à repérer dans beaucoup d'autres dessins, comme par exemple celui-ci (4), où il est suggéré qu'il y a des ressemblances entre une femme vêtue de burqa et la boîte noire d'un avion :

5 La photo est accompagnée du texte suivant : « Il paraît qu'elle s'est vexée car quelqu'un lui a dit qu'elle avait trois beaux enfants !!! ». 


\section{ÉmOI DANS Le SinAi:}

LES RUSSES RETROUVENT UNE BOITT NOIRE

CORROBORANT L'HYPOTHËSE TERRORISTE

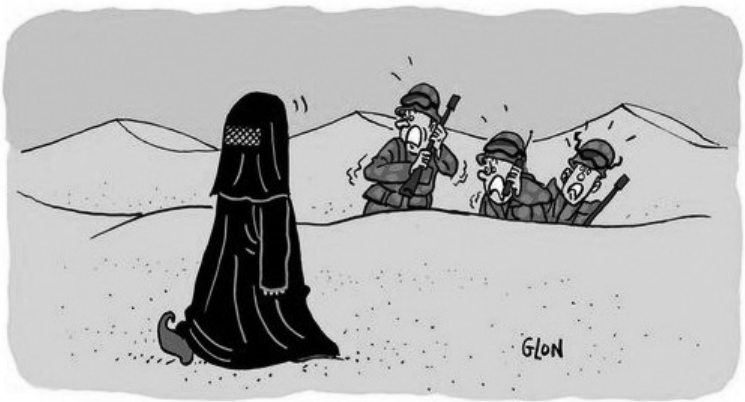

Image 4. Auteur : François Glon

Source : https://twitter.com/glondessins/media

L'acte humoristique de tous ces messages s'appuie sur l'incohérence insolite (terme forgé par Charaudeau, 2006) qui établit une relation entre deux univers étrangers l'un à l'autre, mais entre lesquels le locuteur trouve toutefois un lien. Mais parfois ce lien n'existe pas du tout, comme sur le dessin polonais $n^{\circ} 5$ où l'on juxtapose deux chiens, dont l'un est Reksio, personnage de dessin animé très populaire en Pologne, et l'autre Burek, un chien en burqa, lequel, à cause de l'immigration maghrébine, est supposé être un chien polonais typique du futur. Ici l'humour vient de l'idée de revêtir un chien d'une burqa, mais la plaisanterie est aussi linguistique, car Burek est en Pologne ce qu'est Médor en France, c'est-à-dire un nom couramment donné aux chiens, au point d'en être devenu presque générique.

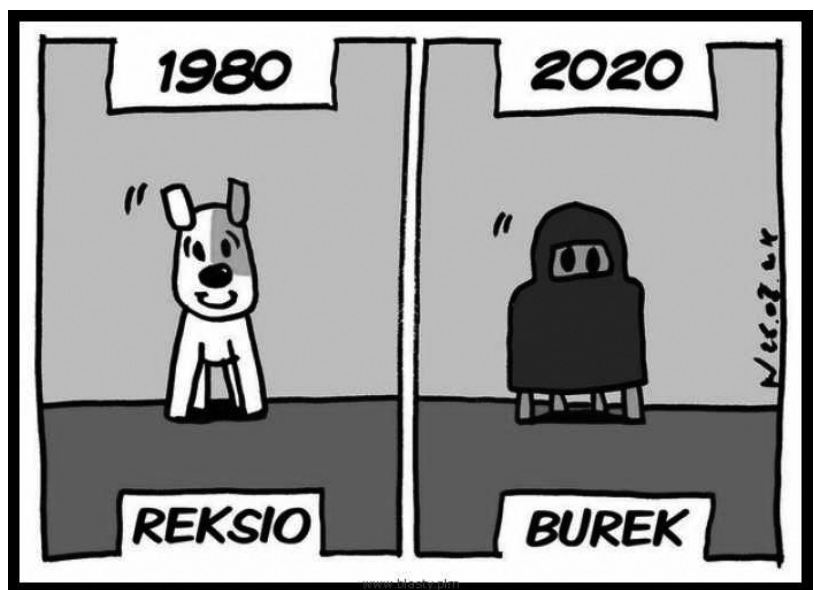

Image 5. Auteur non identifié

Source : https://demotywatory.pl/4777088/Postep 
En général, la burqa est censée rabaisser les femmes musulmanes pour diverses raisons, comme par exemple dans le mème ${ }^{\circ} 6$ où le texte qui canalise le sens de la photo suggère qu'une musulmane est identique à toutes les autres ${ }^{6}$ :

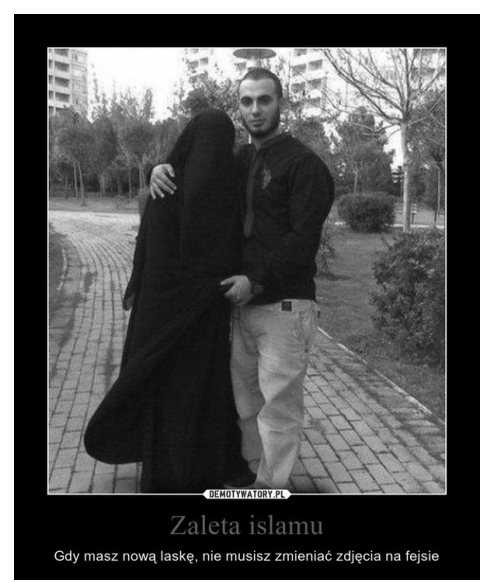

Image 6. Auteur inconnu

Source : https://demotywatory.pl/4650831/Zaleta-islamu

\section{2. La barbe}

Parmi les éléments emblématiques de l'islam tournés en dérision, figure aussi la barbe que les musulmans sont censés devoir porter. Ce symbole est raillé sur le dessin suivant (7) où la barbe islamique est rapprochée de manière insolite de la crête iroquoise des punks dont le port n'est vu que comme un indice de la crise d'adolescence, ce qui suggère que les jeunes recrues djihadistes seraient des jouvenceaux impulsifs, écervelés, qui se livrent à des actions irréfléchies, leur ôtant tout caractère sérieux :

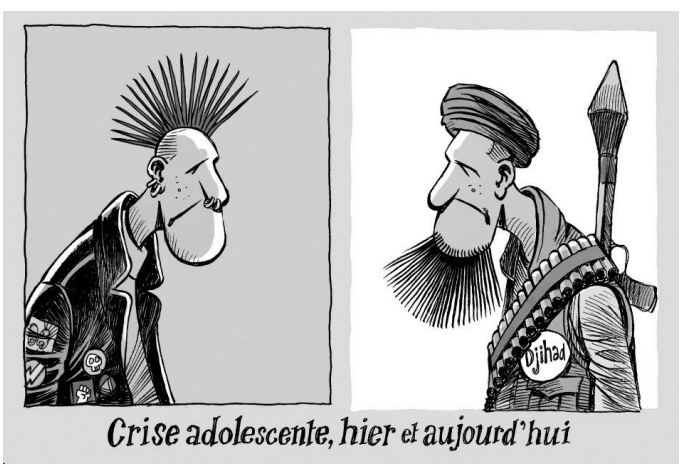

Image 7. Auteur non identifié

Source : https://www.chappatte.com/images/jeunes-djihadistes-europeens/

6 «L'avantage de l'islam : quand t'as une nouvelle gonzesse, mais que tu dois même pas changer ta photo de profil sur Face[book] ». 
La même idée est exprimée dans beaucoup d'autres messages, comme p. ex. celui-ci (8) :

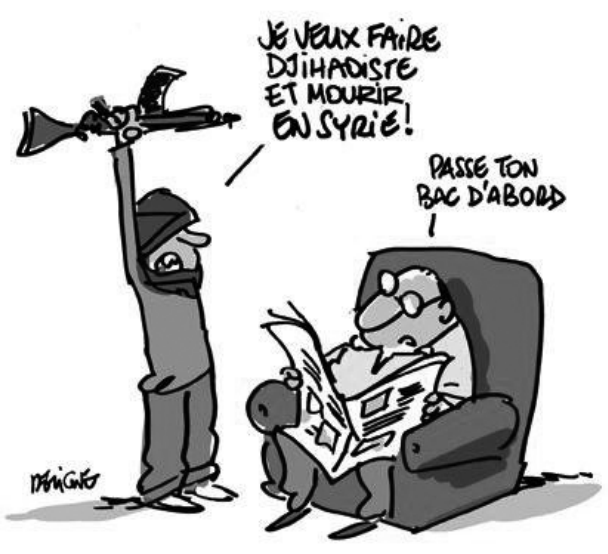

Image 8. Auteur : Frédéric Deligne

Source : https://twitter.com/fdeligne

L'autre exemple où l'on s'en prend à la barbe en tant que symbole islamique, encore plus insolite et basé cette fois-ci sur un matériel typiquement verbal, est le mème $\mathrm{n}^{\circ}$ 10 qui parodie la popstar américaine Justin Bieber, en le décorant d'une barbe et en modifiant son nom en Bomber, nom évoquant la bombe et connotant les djihadistes :

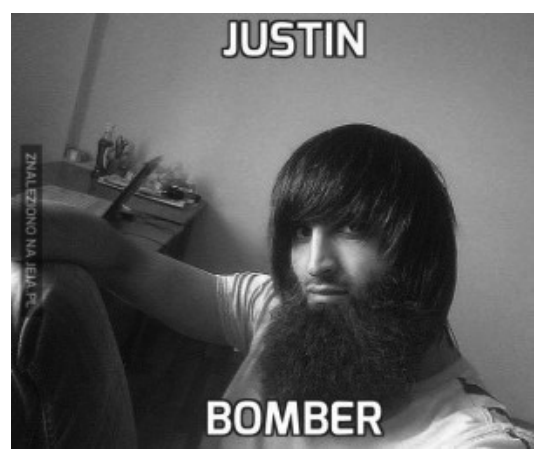

Image 9. Auteur inconnu

Source : https://memy.jeja.pl/159920,justin.html

\section{3. La formule Allahu akbar}

L'autre emblème islamique brocardé dans cette guerre discursive est la formule $\mathrm{Al}$ lahu akbar qui, dans l'islam, est une magnification de Dieu signifiant « Dieu est (le) plus grand », et que les islamistes ont adoptée comme cri de guerre visant à terrifier l'ennemi. Cette formule connote une attaque terroriste, un suicide par explosion, et finalement, par contiguité l'explosion elle-même, ce qui se voit sur le mème numérique 
suivant (10) qui amuse le destinataire par son caractère insolite et loufoque en faisant se télescoper deux réalités incompatibles, l'une sérieuse (l'islamisme) et l'autre légère, quotidienne (du maïs à popcorn) :

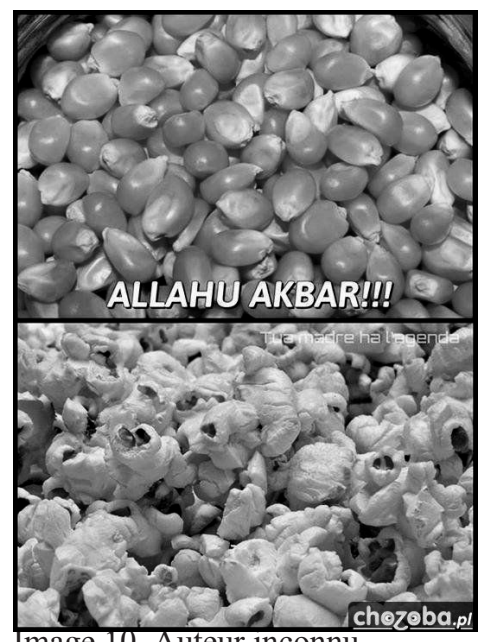

mage 10. Auteur inconnu

Source : https://chozoba.pl/obrazek/1011/rejestracja

La formule Allahu akbar se voit aussi moquée dans les messages se nourissant du mécanisme d'exagération loufoque, tels que le suivant (11), où un policier suggère qu'une vieille dame, dont la voiture a heurté un panneau de limitation de vitesse, est une dhijadiste :

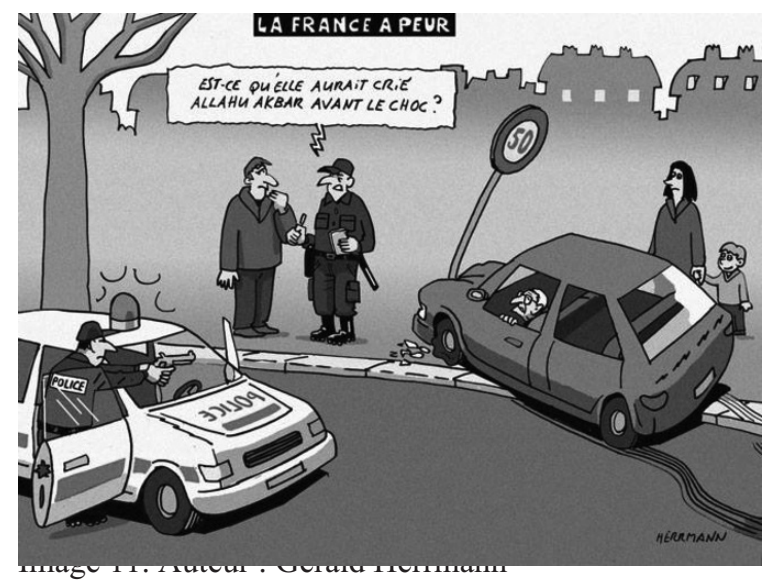

Source : https://www.courrierinternational.com/dessin/2014/12/23/tous-les-chauffards-sont-ilsdes-islamistes 
Ce télescopage de deux réalités permettant au locuteur de redistribuer les valeurs d'une manière insolite, en exploitant ici le mécanisme du contraste, peut s'apprécier dans le mème polonais suivant (12), où un vieux sage chinois demande : "Toutes les places dans l'autobus sont occupées? » et conseille tout bonnement : «Criez Allah akbar !» :

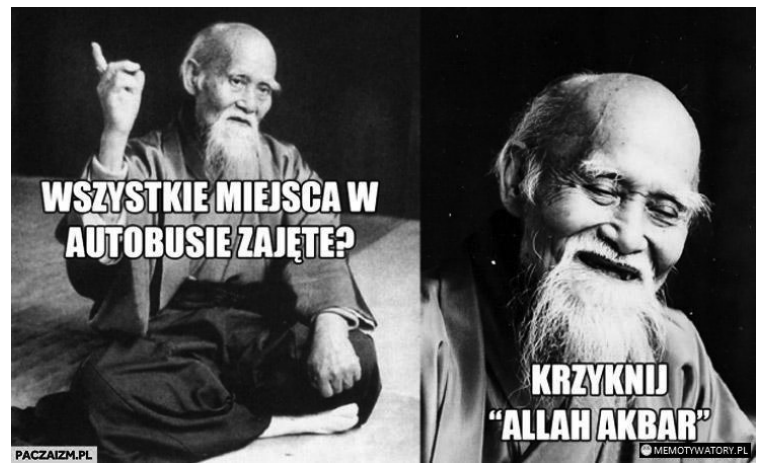

Image 12. Auteur inconnu

Source : https://memotywatory.pl/433550,wszystkie-miejsca-w-autobusie-zajete-krzyknij-allah-akbar

\section{4. Les 72 vierges au paradis}

Un autre élément ciblé par le discours anti-djihadiste est la pratique islamiste de l'attentat-suicide pour tuer les « infidèles » et être récompensé par 72 vierges au paradis. Cette croyance des islamistes est impitoyablement raillée, ce que l'on observe dans le dessin $\mathrm{n}^{\circ} 13$ qui présente un renversement de l'ordre et des valeurs, parce que cette promesse, précieuse pour les djihadistes, est tournée en dérision à travers l'idée que Diesel, la célèbre chienne du Raid qui a perdu la vie au cours d'une opération antiterroriste à Saint-Denis en 2015, rencontre 72 chiens après sa mort en martyr :

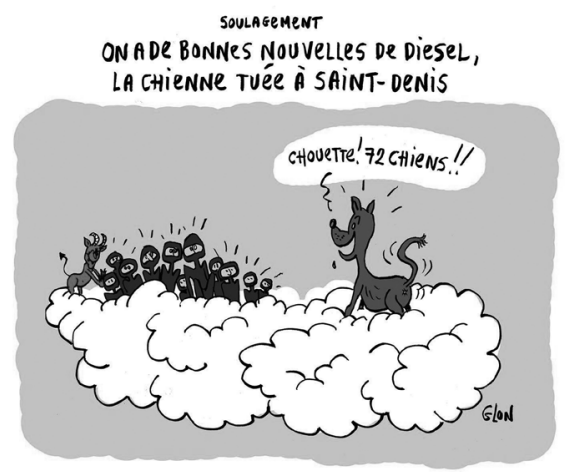

Image 13. Auteur : François Glon

Source : https://twitter.com/glondessins/media 
Mais le comique ne s'arrête pas là, car à y regarder de plus près, ce que la chienne appelle les « chiens », ce sont les djihadistes qui se sont eux-mêmes suicidés.

Cette croyance est également ridiculisée par l'absurde dans le message suivant (14) où les 72 vierges deviennent un « salaire » qui peut être réduit de $50 \%$, ce qui donne quelque chose de totalement insensé :

\section{DAESH REDUIT LE SALATRE DE SES DTIHADISTES!}

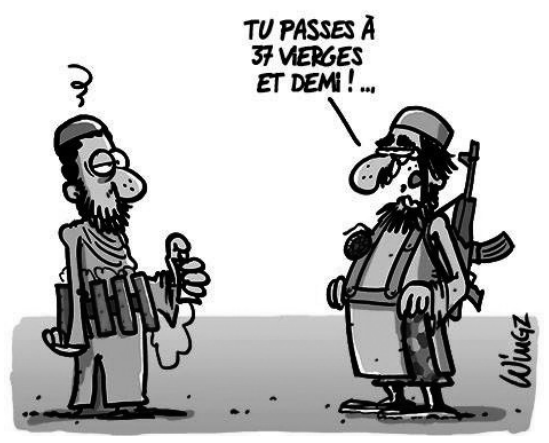

Image 14. Auteur : Benjamin Wingz

Source : http://www.wingz.fr/?s=djihad

\section{5. Les défauts des dhijadistes}

Le discours des internautes ne combat pas seulement les emblèmes islamiques. Sans faire d'amalgame entre musulman et djihadiste, on raille les traits de caractère et les défauts attribués aux islamistes, en particulier l'ignorance (15) et l'hypocrisie (16) :

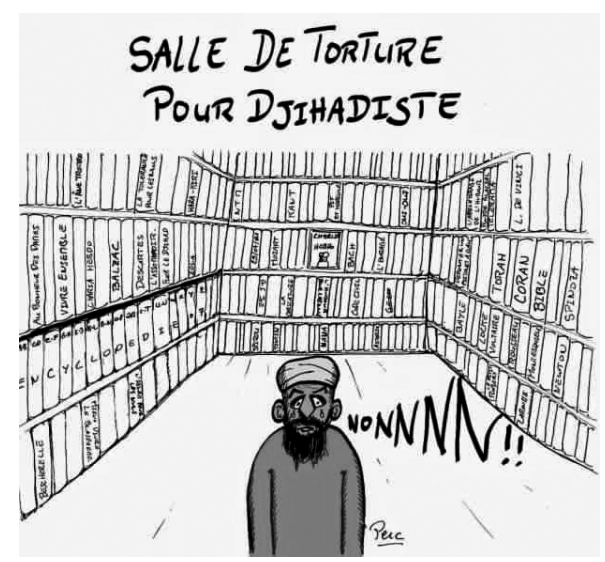

Image 15. Auteur : Perc

Source : https://percdessinpresse.wordpress.com/author/perc317/page/3/ 


\section{LES DJIHADISTES CONTINUENT DE RECRUTER SUR INTERNET}

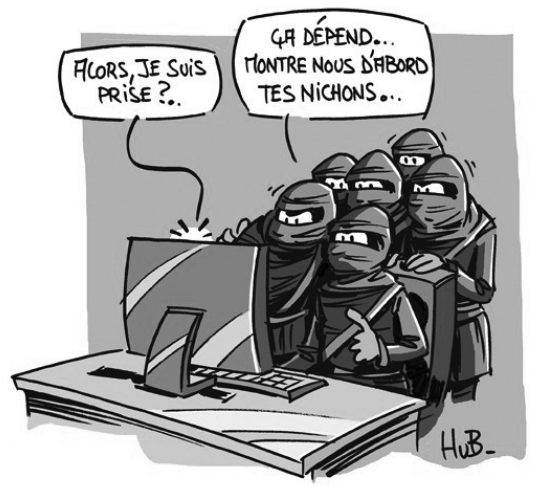

Image 16. Auteur : Humbert Chabuel (Hub)

Source : http://hublog.canalblog.com/archives/2014/09/16/30599255.html

\section{ANALYSE DES MESSAGES POLARISÉS SUR LE SOI}

Il est temps de passer au deuxième type de messages, à savoir ceux où le locuteur focalise son discours sur lui-même et se construit explicitement l'éthos de quelqu'un de totalement différent de son ennemi. On observe cette fois le recours aux emblèmes de la culture du locuteur.

Ainsi, les attaques djihadistes provoquent p. ex. l'apparition des messages comme (17) où l'on fait implicitement référence à la fable de La Fontaine où le chêne, arrogant, est grand, massif et fort, et le roseau, fin et petit, à l'allure chétive, « plie mais ne rompt pas » :

\section{AVis AOX DjiHADistes}

COMME LE FRANQAiS, LE BELGE PLIE MAIS NE ROMPT PAS!

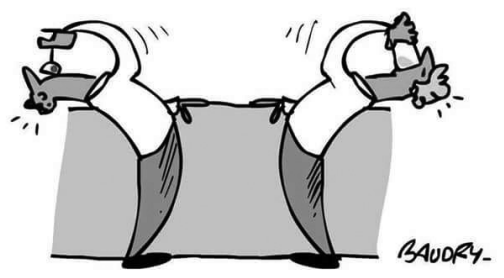

Image 17. Auteur : Hervé Baudry

Source : https://toutelaculture.com/wp-content/uploads/2016/03/Baudry.jpeg 
Ce dessin est une réaction aux attentats terroristes perpétrés en Belgique en 2016. Cette fois-ci, ce qui fait de ce message un acte humoristique, c'est évidemment la transposition du motif littéraire dans un domaine de la vie quotidienne très ordinaire, celui du stéréotype des Français et des Belges buvant du vin et de la bière. La même thématique s'observe dans le dessin $n^{\circ} 18$ :

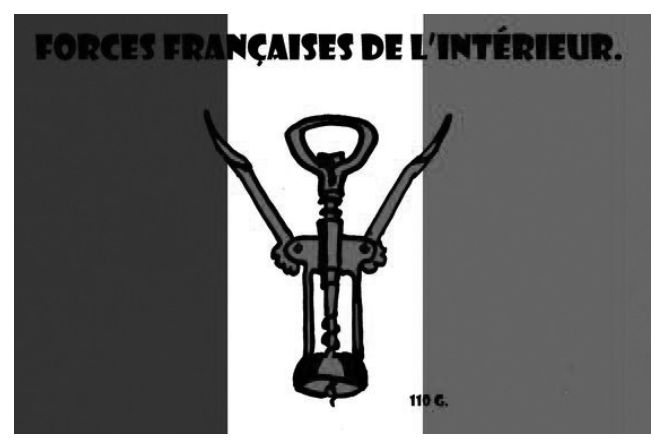

Image 18. Auteur non identifié

Source : https://www.pinterest.fr/pin/786652259892209809/

Ce message aux connotations historiques fait référence aux FFI, groupements militaires de la Résistance pendant la seconde Guerre mondiale. Le symbole de ces « forces » est ici un ouvre-bouteille qui connote le vin et la joie de vivre que le locuteur déclare intacte en dépit des attentats. Mais les « bras » de l'ouvre-bouteille en haut évoquent aussi le général De Gaulle en mai 1968 , moqué pour avoir qualifié les événements de Mai 68 de « chienlit », ce qui lui a valu une réponse des étudiants et des artistes de l'«atelier populaire» de l'École des Beaux-Arts de Paris, alors occupée, sous la forme de l'affiche suivante (19) :

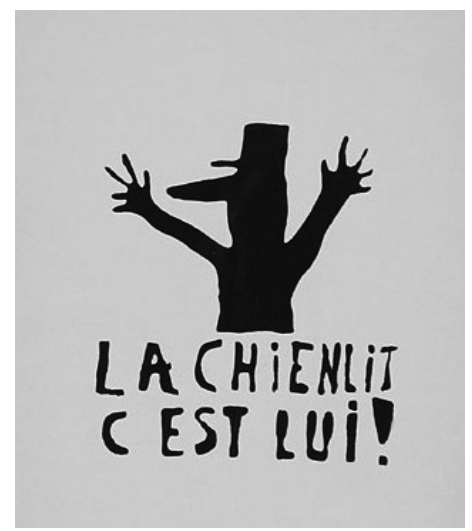

Image 19. Auteur : Atelier populaire

Source : http://www.iisg.nl/collections/may68-posters/d13-244.php

7 Nous remercions le professeur Jean-Pierre Goudaillier pour cette suggestion précieuse. 
Le chef de l'État, levant les mains en l'air en posture victorieuse, est ici présenté plutôt comme une marionnette, et si l'on interprète les bras de l'ouvre-bouteille du message $n^{\circ} 18$ par le biais de cette affiche, on comprend le message destiné aux dhijadistes : peut-être est-ce la chienlit, mais nous restons unis face à l'ennemi commun.

Par ailleurs, l'appel à se relever après les attentats est présent dans beaucoup d'autres messages de notre corpus où il est fait référence à la culture et les coutumes des Français ou des Belges, comme sur le dessin $\mathrm{n}^{\circ} 20$ :

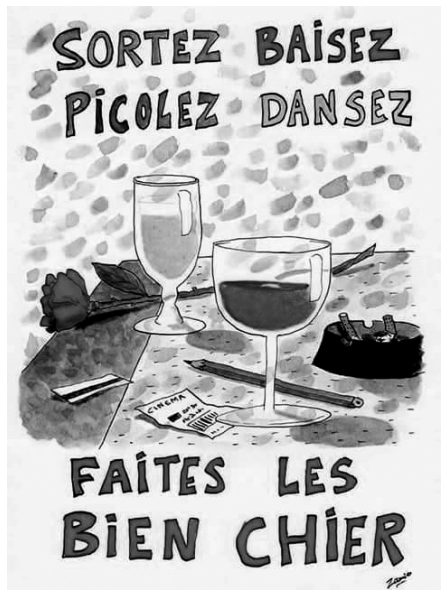

Image 20. Auteur non identifié

Source : http://informationsecteares.overblog.com/2015/11/ce-soir-vendredi-27-novembre-2015.html

ou encore le message $\mathrm{n}^{\circ} 21$, où l'on joue sur la polysémie du mot canon :

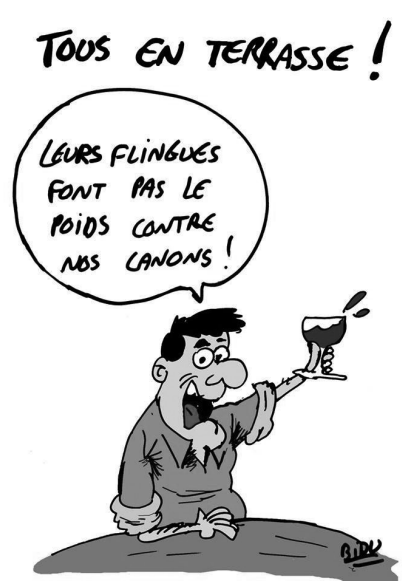

Image 21. Auteur : Olivier Descombes (Bidu)

Source : https://toutelaculture.com/actu/politique-culturelle/quelques-dessins-de-resilience2-ans-apres-les-attentats-de-paris-du-13-novembre-2015/ 
Pour en rester dans cette convention de la joie de vivre, voilà encore deux messages s'appuyant sur les habitudes stéréotypées concernant cette fois-ci la nourriture : l'un qui se base sur l'ironie (23) :

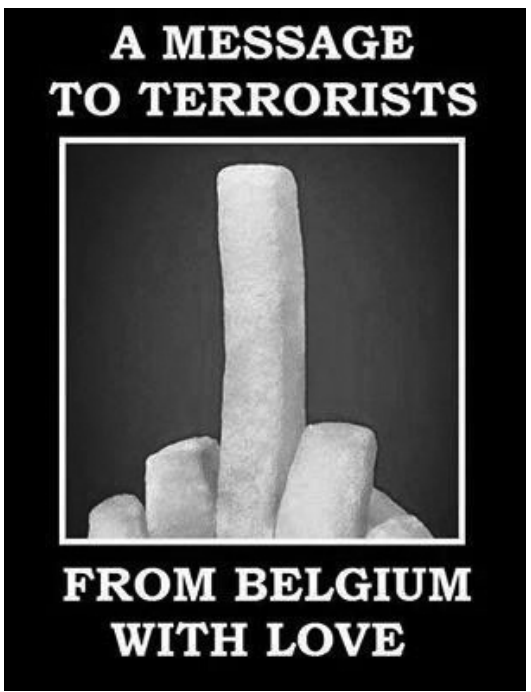

Image 22. Auteur inconnu

Source : https://pl.pinterest.com/pin/130393351690205916/

et l'autre (23), où des internautes belges exploitent le stéréotype français selon lequel les Belges seraient des mangeurs de frites et en font leur emblème et leur arme, ce qui témoigne de leur sérénité et de ce qu'il ne sera pas facile à l'ennemi islamiste de briser leur moral :

\section{Ta mitraillette}

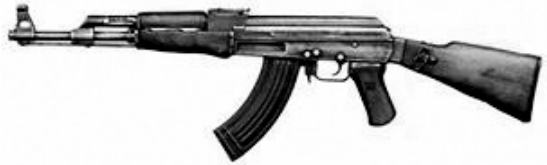

viens te battre!

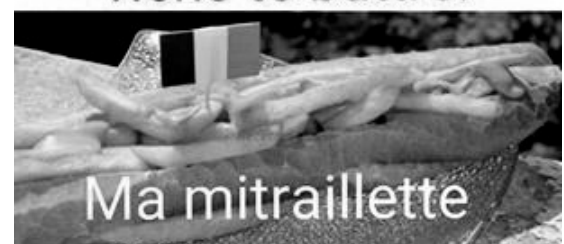

Image 23. Auteur inconnu

Source : https://www.pinterest.com/pin/458593174537842539/ 
Pour en citer d'autres messages imprégnés d'autodérision, mais qui n'affectent en rien le sentiment communautaire des locuteurs - tout au contraire, ils le renforcent - en voilà un faisant référence à l'un des éléments de la culture populaire française : le personnage de Nabilla Benattia, mannequin considérée, à tort ou à raison, comme « stupide » et dont les propos sont le plus souvent raillés (24):

\section{NABILA DANS 5 ANS CONVERTIE AU SALAFISME}

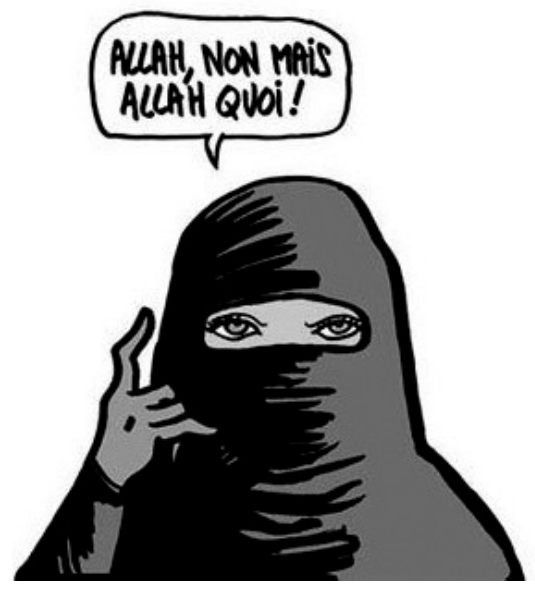

Image 24. Auteur : Ghib

Source : http://ghib.over-blog.com/2014/10/humour-hallal-burqua.html

Le fameux Allô, non mais, allô quoi du mannequin, modifié par recours à la quasi-homophonie insolite en Allah, non mais Allah quoi, ridiculisant le Dieu de l'islam d'une part, a pour fonction, d'autre part, de souder la communauté, tout comme tous les messages où est construit explicitement l'éthos du locuteur.

\section{REGISTRE FAMILIER, POPULAIRE, ARGOT COMMUN : QUELLE(S) FONCTION(S) ?}

Dans les messages analysés transparaît l'éthos discursif du locuteur qui s'y présente comme quelqu'un dont il n'est pas facile de briser le moral, qui aime la vie et sait en profiter, en dépit du danger que constituent la présence et les actes de l'Autre-terroriste. Cet éthos est parfois dit et toujours montré ${ }^{8}$ par la manière de dire, surtout par le ton humoristique adopté : le Soi a certes peur, mais il est capable d'en rire. Mais, dans les messages du premier type, focalisés sur la cible du discours, le Soi est lui aussi présent : il se profile derrière l'Autre, car, comme le remarque Charaudeau (2007), « Tout jugement sur l'autre est en même temps révélateur de soi : il dit peut-être quelque chose de déviant sur l'autre, mais il dit en même temps quelque chose de vrai sur celui qui porte ce jugement ».

8 Voir D. Maingueneau (p. ex. 1999, 2002, 2010). 
Il existe aussi des messages où le locuteur se juxtapose à l'Autre. On voit alors clairement qu'il parle au nom du groupe des « siens », un groupe dont les membres sont liés par des liens identitaires forts. Un exemple intéressant de ce type de messages est fourni par le dessin $n^{\circ} 26$ où deux djihadistes s'énervent en découvrant que l'entaille faite à l'épée à un crayon révèle un " sourire » triomphant. On sait que le crayon est devenu, après l'attaque terroriste contre les journalistes de Charlie Hebdo en janvier 2015, le symbole de la liberté d'expression - valeur chère aux Français - avant de symboliser, par métonymie, la nation française :

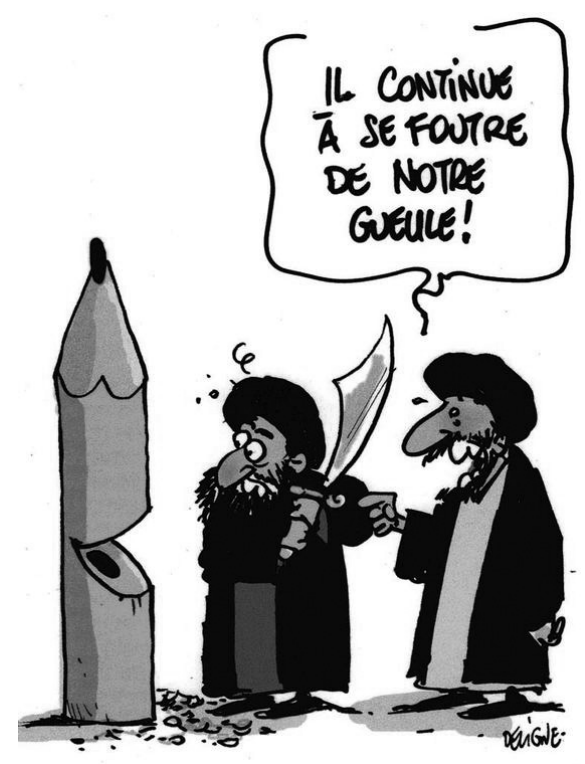

Image 25. Auteur : Frédéric Deligne

Source : https://www.facebook.com/fredericDeligne/photos

L'esprit communautaire transparaissant sur ce dessin est un élément que tous les messages analysés ont pour but de protéger et de renforcer. Moquer les emblèmes de l'Autre en les comparant aux siens, appeler à rire ensemble malgré l'angoisse et la douleur, faire constamment référence aux symboles de son identité collective : tous ces procédés sont employés dans le but de souder le groupe et élaborent un discours identitaire.

L'emploi de termes du registre familier, populaire et plus encore, argotique, a également pour but d'atteindre cette finalité primordiale du discours anti-djihadiste. Nous avons vu plusieurs exemples de messages formulés avec des éléments grossiers : merde, faire chier, pl. laska ('gonzesse'), nichons, baiser, picoler, se foutre de la gueule... il va sans dire que les mots de ce type sont très expressifs, amusent le destinataire et lui épargnent de cette manière un pathos indésirable. Or il semble que leur fonction essentielle soit de combler le besoin de solidarité entre les membres d'une même communauté qui certes, se différencient socialement, mais veulent constituer à l'extérieur un 
groupe fortement uni face à l'ennemi commun. Et puisque l'emploi de ces mots permet d'instaurer une connivence ludique entre le locuteur et le destinataire, nous croyons que l'on peut parler de la fonction grégaire des termes familiers et populaires dans le discours analysé. Ils constituent un code complice remplissant cette fonction de connivence pour des enjeux identitaires, une fonction intégrante. En recourant aux mots des registres familier et populaire et relevant de l'argot, on fait un clin d'œil linguistique à son destinataire.

\section{CONCLUSIONS}

Plusieurs mots-clés s'avèrent fondamentaux pour l'analyse du discours anti-djihadiste des internautes. Puisque l'un des enjeux principaux consiste à attirer des alliés, il obtient la connivence grâce à l'humour. À l'aide du discours humoristique, le locuteur conquiert des complices en flattant leur intelligence car, comme le précise Charaudeau (2006 : 40), «l'auteur d'un acte humoristique se montre intelligent et l'autre en montrant qu'il apprécie, fait preuve à son tour d'intelligence ». En tant que phénomène permettant de porter un regard distancié sur une réalité angoissante et triste, l'humour a en plus une fonction libératrice et cathartique. Il rend supportable l'insupportable et donne ainsi au locuteur la supériorité qu'il n'avait pas. L'humour est incontestablement un facteur de connivence : il crée un lien entre le locuteur et le destinataire, lesquels, en partageant un certain nombre de références communes, s'allient contre la cible du discours humoristique qui remplit alors une fonction identitaire et unificatrice, tout comme le recours aux registres de langue considérés comme " bas », employés entre les pairs.

S'attirer des alliés, avons-nous dit. Dans quel but ? Pour mieux faire face à l'ennemi, même s'il ne s'agit que d'une lutte discursive. Un ennemi qui tue non seulement physiquement, mais aussi moralement. Pour qu'il n'y parvienne pas, il faut se renforcer. Tous les messages du deuxième groupe, polarisés autour de la construction de l'éthos du locuteur qui lutte contre la cible de son discours en se montrant résistant aux attaques, ont pour but de montrer à l'ennemi que l'on est différent de lui et, par là, meilleur. Si le Soi s'affiche comme sachant profiter de la vie, c'est que l'ennemi est supposé être un arriéré qui se suicide pour des chimères du type " 72 vierges après la mort », si le Soi est capable d'autodérision, c'est qu'il est intelligent et sait prendre ses distances par rapport à sa communauté, avec laquelle il reste toutefois fortement lié. On l'aura $\mathrm{vu}$, le locuteur construit son éthos non seulement de façon directe dans les messages de la deuxième catégorie où il s'autoprésente, mais aussi indirectement dans les messages de la première catégorie, où il attire l'attention du destinataire sur ce qu'est l'Autre, laissant par là entrevoir ce que lui-même n'est sûrement pas.

Paradoxalement, cet Autre, bien qu'il soit un ennemi, est indispensable au Soi. Comme l'élucide Sophie Moirand, « la conscience de l'identité passe par le ressenti de l'altérité » (2015:12). Et s'il est vrai que le discours anti-dijadiste analysé a pour objectif de railler les extrémistes, il s'en profile un autre encore en profondeur, beaucoup plus important : celui d'unir le groupe, de le souder, de renforcer les liens qui existent entre ses membres qui, en ces temps d'incertitude, ont besoin d'affirmer leur propre identité. 


\section{Bibliographie}

AMOSSY, Ruth ([2000] 2006) L'Argumentation dans le discours. Paris: Colin.

AMOSSY, Ruth (2011) « Des sciences du langage aux science sociales : l'argumentation dans le discours. » A contrario 16, 10-25.

BONENFANT, Maude (2014) «Le mème numérique : étude sémiotique des réseaux à partir des concepts de trace et d'indice. » RISCP. http://communiquer.revues.org/1295 ; DOI : 10.4000/communiquer.1295.

CHARAUDEAU, Patrick (2006) « Des Catégories pour l'Humour? » Questions de communication 10, 19-41.

CHARAUDEAU, Patrick (2007) «Les stéréotypes, c'est bien. Les imaginaires, c'est mieux. » In : H. Boyer (éd.), Stéréotypage, stéréotypes : fonctionnements ordinaires et mises en scène. Vol. 4. Paris : L'Harmattan, 47-63. http://www.patrick-charaudeau. com/Les-stereotypes-c-est-bien-Les, $98 . \mathrm{html}$

CHARAUDEAU, Patrick (2009) «L'identité culturelle entre soi et l'autre. » In : Actes du colloque de Louvain-la-Neuve en 2005, http://www.patrick-charaudeau.com/L-identiteculturelle-entre-soi-et.html

DAWKINS, Richard (1976) The Selfish Gene. Oxford: Oxford University Press.

JAKOBSON, Roman (1971) « Language in relation to other communication systems. » In : R. Jakobson, Selected Writings 2, Word and Language. The Hague: Mouton, 697-708.

MAINGUENEAU, Dominique (1999) « Ethos, scénographie, incorporation. » In : R. Amossy (éd.), Images de soi dans le discours - La construction de l'ethos. Lausanne : Delachaux et Niestlé, 75-100.

MAINGUENEAU, Dominique (2002) «L'éthos, de la rhétorique à l'analyse du discours. » [Version raccourcie et légèrement modifiée de "Problèmes d'éthos »]. Pratiques 113114), http://dominique.maingueneau.pagesperso-orange.fr/pdf/Ethos.pdf

MAINGUENEAU, Dominique (2010) «Éthos dit, éthos montré. » In : J. Górnikiewicz/H. Grzmil-Tylutki/I. Piechnik (éds.), En quête de sens. W poszukiwaniu znaczeń. Études dédiées à Marcela Świqtkowska. Studia dedykowane Marceli Światkowskiej. Cracovie : Éditions de l'Université Jagellonne, 294-301.

MOIRAND, Sophie Colette (2015) «Préface». In : N. Guelli1/F. Hailon/A. Richard (éds.), Le discours politique identitaire dans les médias. Paris : L’Harmattan, 9-14.

\section{Résumé}

\section{LA CONSTRUCTION DU DISCOURS ANTI-DJIHADISTE SUR INTERNET : ENJEUX, THÉMATIQUES, PROCÉDÉS}

Cet article analyse certains aspects particuliers de la construction du discours antidjihadiste d'internautes européens qui semblent avoir déclaré une guerre ouverte aux terroristes islamistes. Se déroulant sur le plan discursif, cette guerre se manifeste sous forme de phénomènes communicationnels divers. Il s'agit ici d'analyser un type de messages spécial, exploitant différents codes sémiotiques (surtout iconique et verbal) qui, de par leur caractère humoristique attrayant, captent l'attention du public 
et acquièrent une grande popularité en peu de temps : les mèmes numériques et les dessins satiriques. Largement relayés sur Internet, ces phénomènes viraux prennent appui sur l'humour. Et puisque tout acte de discours humoristique est un acte d'énonciation à des fins stratégiques pour faire de son interlocuteur un complice, ces messages sont d'excellents outils de lutte discursive contre la propagande et les actes djihadistes. Ils sont susceptibles de produire sur l'auditoire un effet de connivence qui entraîne l'adhésion du destinataire à la disqualification de la cible du discours. L'article décrit la façon dont s'effectue cette disqualification de l'image du djihad et de l'islam en général, ainsi que le matériel verbal et iconique exploités à cette fin. On s'intéressera aux principes et aux procédés de construction du discours en question, à ses effets humoristiques dans le contexte de son apparition, de même qu'à sa finalité et sa thématique.

Mots-clés : discours anti-djihadiste, islam, énonciation, Internet, humour

\section{Abstract \\ THE CONSTRUCTION OF THE ANTI-JIHADIST DISCOURSE ON THE INTERNET: CHALLENGES, THEMES, METHODS}

The article analyses some aspects of the construction of the anti-jihadist discourse of European internet users who seem to have declared an open war against Islamist terrorists. This war is taking place on the discursive level, and it manifests itself in the form of various communication phenomena. The aim of the paper is to analyse a special kind of messages, exploiting various semiotic codes (especially visual and verbal), which attract the attention of audiences and acquire great popularity within a short time: digital memes and speech bubbles. These viral phenomena, widespread on the internet, use humour as their main tool. And since every act of humorous discourse is an act of enunciation whose strategic purpose is to turn its interlocutor into an accomplice, these messages are excellent means of discursive struggle against propaganda and jihadist acts. They are likely to produce in the audience an effect of connivance, leading the recipient to adhere to the disqualification of the target of the speech. The article describes how this disqualification enables one to attack the image of jihad and Islam in general, as well as the verbal and visual material exploited for this purpose. It also focuses on the principles and processes of the construction of the discourse in question, its humorous effects, as well as its aims and themes.

Keywords: anti-jihadist speech, Islam, enunciation, internet, humour 


\section{Povzetek \\ OBLIKOVANJE PROTIDŽIHADISTIČNEGA DISKURZA NA INTERNETU: VLOGA, TEMATIKE, POSTOPKI}

V članku analiziramo nekatere posebne vidike oblikovanja protidžihadističnega diskurza v objavah evropskih internetnih uporabnikov, za katere se zdi, da so odprto napovedali vojno islamskim teroristom. Ta vojna, ki poteka na diskurzivni ravni, se kaže v mnoštvu različnih komunikacijskih pojavov. Raziskujemo posebno vrsto sporočil, ki uporabljajo različne semiotične kode (predvsem slikovne in besedne), ki s privlačnim humornim slogom pritegnejo pozornost bralcev in postanejo v kratkem času zelo priljubljeni. Gre za numerične meme in satirične karikature. So množično prisotni na internetu, kjer jim je omogočeno hitro širjenje, in se opirajo zlasti na humor. In ker ima vsako humorno diskurzivno dejanje za cilj, da sogovornika spremeni v sostorilca, so ta sporočila odlična orodja za besedni boj proti džihadistični propagandi in dejanjem. Z bralci se lahko vzpostavi občutek povezanosti, ki naslovnika pritegne k obsojanju ciljev diskurza. V članku opišemo način, kako se razvije obsojanje podobe džihada in islama, pa tudi jezikovna in slikovna sredstva, ki temu namenu služijo. Zanimamo se torej za načela in postopke oblikovanja takšnega diskurza, za humorne učinke, ki spremljajo njihovo pojavitev, pa tudi za funkcijo in tematiko tega diskurza.

Ključne besede: protidžihadistični govor, islam, izrekanje, internet, humor 